\title{
NOUVELL
}

\section{Les enzymes qui contrôlent la torsion de I'ADN}

Nynke H. Dekker, Thierry Viard, Claire Bouthier de La Tour, David Bensimon, Vincent Croquette

> Notre expérience quotidienne montre qu'il est impossible de désenchevêtrer une pelote de ficelle sans faire de nœuds. Le noyau cellulaire est lui-même une fantastique pelote de chromosomes. Chacun de nos chromosomes mesure quelques centimètres de long et se trouve enchevêtré dans un noyau de quelques millièmes de centimètres. Cependant, lors de la division cellulaire, les 46 chromosomes de chaque cellule-mère arrivent à se séparer sans former de nœuds pour être distribués à part égale dans chacune des cellules-filles. Le mécanisme de ce petit miracle n'est que partiellement compris.

On sait que des enzymes - les topo-isomérases - sont essentielles pour désenchevêtrer l'ADN chromosomique, mais le scénario précis de leur action n'est toujours pas bien connu. L'implication des topo-isomérases dans les processus de réplication, de transcription et de recombinaison, où la topologie de l'ADN joue également un rôle significatif, souligne combien il est important de comprendre le mécanisme d'action de cette famille d'enzymes [1]. Leur inhibition conduisant à la mort cellulaire, ces enzymes sont la cible de médicaments anti-cancéreux et anti-bactériens [2].

Il existe deux sortes de topo-isomérases, baptisées Topo I et Topo II selon qu'elles coupent transitoirement un seul ou les deux brins de l'ADN respectivement. Ainsi, les Topo I, dont il est question dans cet article, agissent en modifiant le nombre d'enlacements des brins à l'intérieur d'une double hélice d'ADN, cependant que les Topo II catalysent le passage d'un segment d'ADN double-brin à travers un autre.

Différents modèles ont été proposés pour le fonctionnement de la Topo I (Figure 1). Dans le premier, l'enzyme, après avoir clivé un premier brin d'ADN, maintient solidement les deux extrémités de la brèche et fait passer l'autre brin à travers cette brèche avant de la refermer (Figure 1A). Ce mécanisme, proposé pour la famille des topo-isomérases IA, permet de réduire la contrainte de torsion exercée sur l'ADN de un tour à chaque cycle enzymatique [3, 4]. Dans le second modèle, après avoir clivé le premier brin d'ADN, l'enzyme reste liée fortement à l'extrémité 3' ainsi produite, cependant que l'autre extrémité de la brèche est libre d'effectuer un nombre indéfini de rotations autour de l'autre brin de I'ADN (Figure 1B). Ce mécanisme, proposé pour la famille des topo-isomérases $\mathrm{IB}$, réduit la contrainte de torsion de plusieurs tours par cycle enzymatique [5]. Néanmoins, il n'y a pas, jusqu'à présent, de preuve formelle en faveur de l'un ou l'autre de ces modèles pour ces deux familles de topo-isomérases. Une approche de ce problème a été tentée par des mesures de cinétique enzymatique classiques dans lesquelles un grand nombre de molécules d'enzymes et d'ADN interagissent. Ces mesures ne donnent accès qu'à des valeurs moyennes et ne permettent pas de trancher entre les deux modèles.

Le développement de stratégies utilisant des molécules uniques - démarche que nous avons utilisée pour déterminer le mécanisme des topo-isomérases
N.H. Dekker: Department of

Nanoscience,

Delft University of

Technology, Lorentzweg 1,

2628 CJ Delft, Pays-Bas.

T. Viard,

C. Bouthier de La Tour:

Laboratoire d'Enzymologie

des Acides Nucléiques,

Institut de Génétique et

Microbiologie,

Cnrs UMR 8621,

Bâtiment 400, Université

Paris Sud, Centre d'Orsay,

91405 Orsay Cedex, France.

D. Bensimon, V. Croquette:

Laboratoire de Physique

Statistique et Département

de Biologie,

École Normale Supérieure,

Cnrs UMR 8550,

24, rue Lhomond,

75231 Paris Cedex 05, France.

nynke.dekker@mb.tn.tudelft.nl

viard@igmors.u-psud.fr

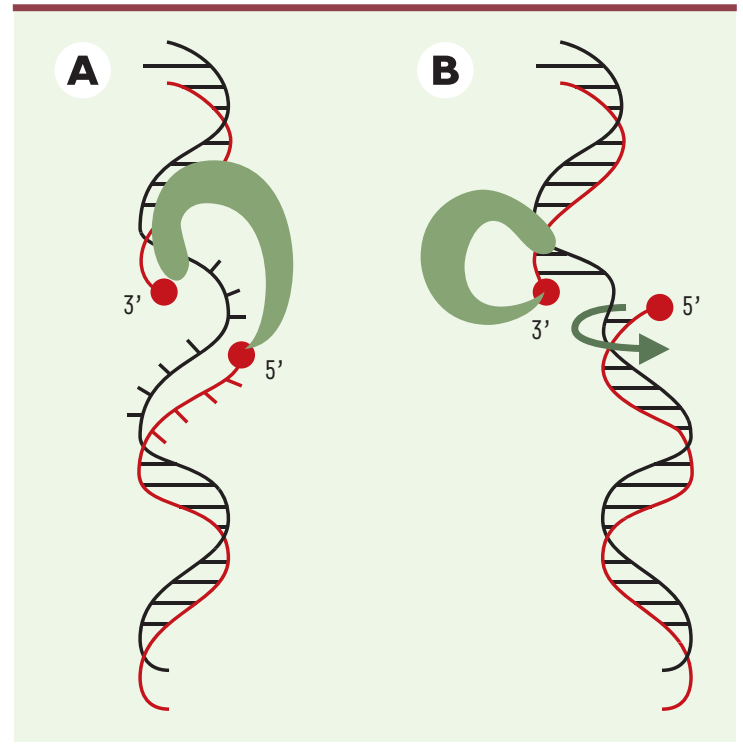

Figure 1. Deux modèles de fonctionnement pour les topo-isomérases I. A. Modèle de passage de brin. La topo-isomérase (figurée par un croissant vert), après avoir clivé l'un des brins (en rouge), maintient les extrémités en place par un pont et laisse passer le second brin (noir) à travers la brèche. $B$. Modèle de rotation libre. Après clivage, la topo-isomérase reste fixée à l'extrémité 3 ' du brin clivé, tandis que l'extrémité 5 ' tourne librement autour de l'autre brin (noir). 


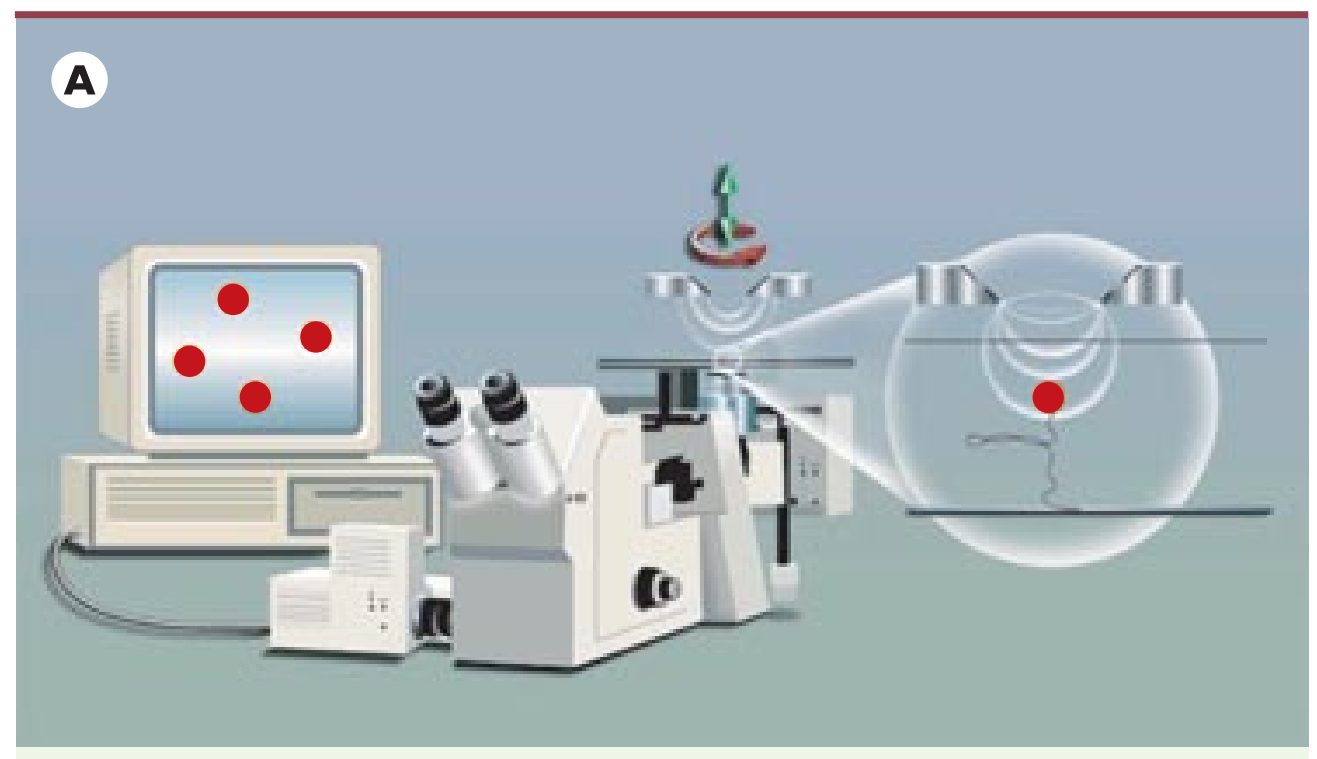

B

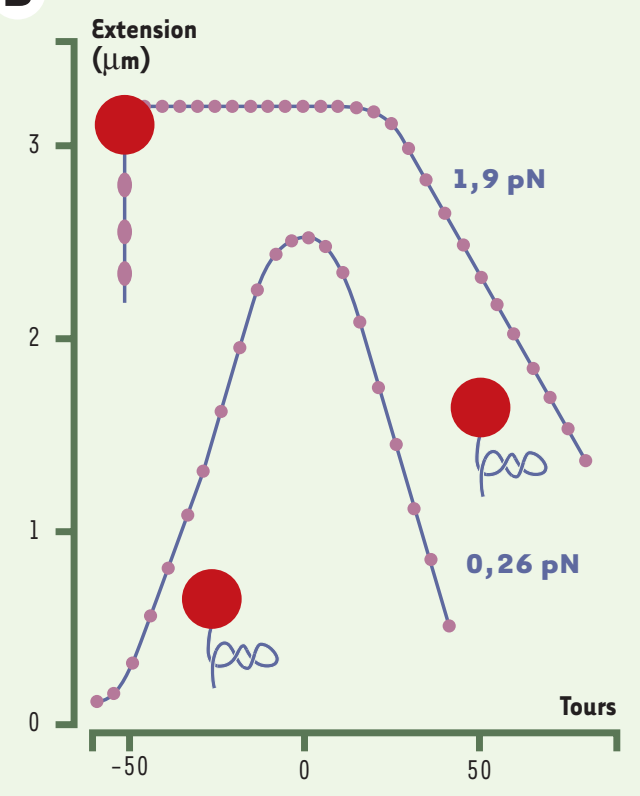

C

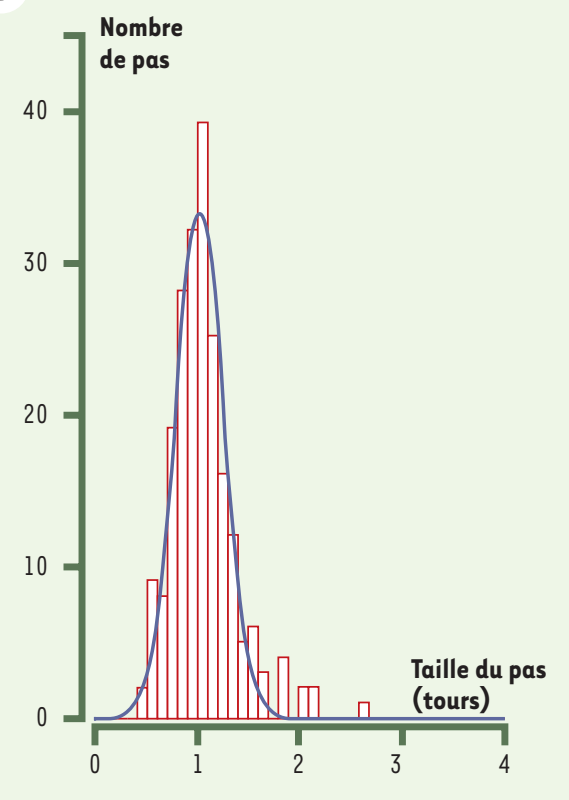

Figure 2. Principe de la mesure de l'extension d'une molécule d'ADN. A. Accrochée d'un côté à une surface en verre et de l'autre côté à une bille magnétique (cercle ro uge) (par des liaisons appropriées), une molécule unique d'ADN forme le substrat dans cette expérience. Au-dessus du capillaire contenant la molécule d'ADN, de petits aimants produisent un gradient de champ magnétique et exercent par conséquent une force sur la molécule d'ADN. Le capillaire lui-même est monté sur un microscope inversé, dont l'image est captée par une caméra CCD. Le mouvement brownien des billes magnétiques et leur distance à la surface du capillaire sont analysés par un logiciel adéquat (image utilisée avec la permission de F. da Cunha). B. L'extension d'une molécule d'ADN double brin de 11500 paires de bases en fonction de la rotation à deux forces différentes. À une faible force $(0,26 \mathrm{pN})$, l'enroulement positif et l'enroulement négatif réduisent tous deux l'extension de la molécule d'ADN à la suite de la formation de plectonèmes. À une force plus élevée $(1,9 \mathrm{pN})$, on note une cassure de symétrie entre l'enroulement positif et négatif. Cette asymétrie est due à la formation de régions non appariées dans l'ADN sous contrainte négative, mais qui n'existent pas dans I'ADN sous contrainte positive. C. Histogramme de l'activité d'une topo-isomérase thermophile sur une molécule d'ADN contenant une région non appariée. Comme le surenroulement n'est relâché qu'en unités d'un tour, on conclut que le modèle du passage de brin est correct.

IA [6] - permet d'une façon générale d'examiner les détails d'interactions biologiques difficiles à appréhender autrement. Ces outils permettent de mesurer très précisément en temps réel l'interaction entre une seule molécule d'enzyme et une molécule unique d'ADN. En ce qui concerne les topo-isomérases I, plusieurs questions se posent: combien de tours la Topo I relâche-telle par cycle? Comment ces enzymes sont-elles activées? De quelle manière la vitesse de «désenroulement » dépend-elle de la force de traction exercée sur l'ADN? Quelle est l'étape limitante du cycle enzymatique?

Pour répondre à certaines de ces questions, nous avons récemment développé un système de micromanipulation et de mesure de force à l'échelle d'une molécule unique dont l'atout principal est la possibilité de contrôler précisément à la fois la force de traction exercée sur I'ADN et surtout le degré d'enroulement de la molécule [7]. Ce dernier paramètre n'est pas contrôlable par d'autres techniques de micromanipulation telles que l'AFM (atomic force microscopy) ou les pinces optiques $(\rightarrow)$.

Dans notre système, une molécule d'ADN est liée à une bille magnétique et à une surface convenable-

$$
\begin{aligned}
& (\rightarrow) m / s \\
& 2003, n^{\circ} 3, \\
& \text { p. } 364
\end{aligned}
$$


ment prétraitée (Figure 2A). Un champ magnétique est produit par deux aimants de NdFeB (neodymium-iron boron) dont les pôles sont très proches l'un de l'autre. La force due au gradient du champ peut être réglée de manière très précise entre 0,1 et 100 picoNewtons $(\mathrm{pN})$ pour des billes magnétiques de 4,5 $\mu \mathrm{m}$ de diamètre. La rotation des aimants, assurée par un moteur, permet d'introduire à volonté une contrainte de torsion d'un nombre de tours donné. Ce système est idéal pour des études sur les topo-isomérases et nous a permis d'observer en temps réel l'activité d'une seule molécule de topo-isomérase sur l'ADN.

L'effet de la rotation sur une molécule unique d'ADN d'une longueur de 11,5 kb est illustré sur la Figure 2B. En l'absence de rotation, la molécule sous faible tension $(0,26 \mathrm{pN})$ a une extension de 2,6 $\mu \mathrm{m}$. À cette force, on s'aperçoit que l'extension de la molécule diminue au fur et à mesure que l'on tourne les aimants, soit vers la droite (torsion positive), soit vers la gauche (torsion négative). Cette décroissance de l'extension de la molécule entraîne la formation de structures surenroulées appelées «plectonèmes » qui correspondent à l'enlacement de I'ADN double-brin sur lui-même. Cette description est une façon savante d'expliquer comment les fils du combiné téléphonique forment des tortillons. La diminution de la taille de la molécule d'ADN, quand on tourne les aimants, nous permet de mesurer avec une grande précision son état de sur-enroulement. En tournant les aimants avec une force plus importante $(1,9 \mathrm{pN})$, une asymétrie apparaît entre les surenroulements positifs et négatifs: les surenroulements négatifs ne conduisent plus à une diminution de la taille de la molécule d'ADN, car, pour une force élevée, ils favorisent plutôt la dénaturation des brins d'ADN.

L'action d'une Topo IA, qui s'accroche préférentiellement à I'ADN simple brin, peut être observeé sur le substrat d'une molécule d'ADN soumise à une torsion négative à une force intermédiaire $(0,7 \mathrm{pN})$. L'ajout d'une topo-isomérase relâche la con- trainte de torsion et ramène l'extension de la molécule d'ADN à son niveau initial en l'absence de rotation. Cette technique nous a permis de mesurer la vitesse de fonctionnement des Topo IA sur I'ADN. Cependant, nous avons montré que plusieurs molécules de ces enzymes se fixent sur l'ADN soumis à une contrainte de torsion négative, car un tel ADN contient plusieurs régions non appariées.

Pour observer l'activité d'une seule molécule de Topo IA sur I'ADN, nous avons donc utilisé un ADN modifié dans lequel une région de 12 nucléotides non appariés a été introduite [8]. Si l'on applique une contrainte de torsion positive, cette région non appariée est la seule qui demeurera à l'état de simplebrin et sa taille est telle qu'une seule molécule de Topo IA pourra s'y fixer.

$\varepsilon$ n utilisant cet ADN substrat, et en ralentissant la vitesse de l'enzyme, nous avons pu observer les pas élémentaires de relaxation de la contrainte de torsion. Nous avons mesuré les tailles de ces pas (convertis en unités de tours) dont la distribution complète est visible sur la Figure 2C. On voit clairement une distribution d'événements centrée autour d'un seul tour, ce qui nous indique que, dans ces conditions, l'enzyme diminue la torsion dans une molécule d'ADN un tour après l'autre tour. Ce résultat correspond à la prédiction faite par le modèle du passage des brins (Figure 1A). De plus, nous avons vérifié ce résultat pour deux topo-isomérases, celle d'Escherichia coli, et celle d'une bactérie thermophile [9], deux organismes bien différenciés au cours de l'évolution.

Avec ce résultat, et sur les bases de données structurales et biochimiques [4, $10,11]$, nous pouvons conclure que le modèle du 12126-31.

passage de brin décrit correctement l'action d'une topo-isomérase IA sur I'ADN. Ce résultat distingue l'action de la Topo IA de celle de la famille IB, dont le mécanisme semble être « rotationnel » et renforce l'idée qu'in vivo la topo-isomérase IA reconnaît et agit sur les régions simplebrin de l'ADN qui apparaissent dans toutes les transactions impliquant la double hélice. $\diamond$

Enzymes that relax supercoiled DNA

\section{REMERCIEMENTS}

Nous adressons nos sincères remerciements au Pr Michel Duguet pour sa lecture critique du manuscrit. Nous remercions l'École Normale Supérieure, le Cnrs, et l'Association de la Recherche sur le Cancer pour leurs financements facilitant cette recherche. N.H. Dekker a bénéficié de l'aide d'une bourse Marie Curie de l'Union européenne et $T$. Viard d'une allocation du ministère de la Recherche.

\section{RÉFÉRENCES}

1. Champoux JJ. DNA topoisomerases: structure, function, and mechanism. Annu Rev Biochem 2001; 70 : 369-413.

2. Pourquier P. De nouveaux rôles pour l'ADN topoisomérase I. Med Sci 2002; 18: 975-81.

3. Brown PO, Cozzarelli NR. A sign inversion mechanism for enzymatic supercoiling of DNA. Science 1979; 206 : 1081-3.

4. Brown PO, Cozzarelli NR. Catenation and knotting of duplex DNA by type I topoisomerases: a mechanistic parallel with type II topoisomerases. Proc Natl Acad Sci USA 1981; 78 : 843-7.

5. Stewart L, Redinbo MR, Qiu $X$, Hol WG, Champoux JJ. A model for the mechanism of human topoisomerase I. Science 1998; 279: 1534-41.

6. Dekker NR, Rybenkov VV, Duguet $M$, et al. The mechanism of type IA topoisomerases. Proc Natl Acad Sci USA 2002; 99 :
7. Strick T, Allemand JF, Bensimon D, Bensimon A, Croquette V. The elasticity of a single supercoiled DNA molecule. Science 1996; 271: 1835 -7.

8. Kirkegaard K, Wang JC. Bacterial DNA topoisomerase I can relax positively supercoiled DNA containing a single-stranded loop. J Mol Biol 1985; 185 : 625-37.

9. Viard T, Lamour V, Duguet M, Bouthier de La Tour C. Hyperthermophilic topoisomerase I from Thermotoga maritima: a very efficient enzyme that functions independently of zinc-binding. J Biol Chem 2001; 276: 46495-503.

10. Li Z, Mondragon A, DiGate RJ. The mechanism of type IA topoisomerase-mediated DNA topological transformations. Mol Cell 2001; 7: 301-7.

11. Changela A, DiGate RJ, Mondragon A. Crystal structure of a complex of a type IA DNA topoisomerase with a single-stranded DNA molecule. Nature 2001 ; 411 : 1077-81. 